\title{
Quality improvement goals for pediatric acute kidney injury: pediatric applications of the 22nd Acute Disease Quality Initiative (ADQI) conference
}

\author{
David T. Selewski ${ }^{1}$ (D) David J. Askenazi ${ }^{2} \cdot$ Kianoush Kashani $^{3} \cdot$ Rajit K. Basu $^{4} \cdot$ Katja M. Gist $^{5} \cdot$ Matthew W. Harer $^{6} \cdot$ \\ Jennifer G. Jetton ${ }^{7}$. Scott M. Sutherland ${ }^{8}$ - Michael Zappitelli ${ }^{9}$. Claudio Ronco ${ }^{10}$. Stuart L. Goldstein ${ }^{11}$. \\ Theresa Ann Mottes ${ }^{12}$
}

Received: 2 June 2020 / Revised: 24 September 2020 / Accepted: 15 October 2020 / Published online: 12 January 2021

(C) IPNA 2021

\section{Introduction}

Acute kidney injury (AKI) is common in hospitalized children and is associated with increased morbidity and mortality across pediatric populations [1-21]. AKI also represents a significant public health burden, substantially increasing healthcare costs across the age spectrum [22-24]. Recent epidemiologic data clearly shows that a large proportion of hospitalized children present with AKI at admission, suggesting that AKI events frequently begin in the community $[1,6]$. As the impact of AKI in children has become clear, it is critical to focus on strategies that improve the process of care by developing care pathways with defined quality metrics/indicators and by disseminating these tools to optimize AKI care to improve outcomes in children at risk of AKI.

David T. Selewski

selewski@musc.edu

1 Department of Pediatric, Medical University of South Carolina, 96 Jonathan Lucas St, CSB 428 MSC 608, Charleston, SC 29425, USA

2 Department of Pediatrics, University of Alabama Birmingham, Birmingham, AL, USA

3 Division of Nephrology and Hypertension, Division of Pulmonary and Critical Care Medicine, Department of Medicine, Mayo Clinic, Rochester, MN, USA

4 Children's Healthcare of Atlanta, Emory University School of Medicine, Atlanta, GA, USA

5 Department of Pediatrics, Children's Hospital of Colorado, University of Colorado Anschutz Medical Campus, Aurora, CO, USA
The 22nd Acute Disease Quality Initiative (ADQI) convened multidisciplinary experts from across the globe in San Diego, California, to provide a framework to improve the quality of care in adults at risk for AKI. Briefly, this group identified and discussed best practices in "prevention, identification, and care of patients with AKI" across the spectrum of healthcare settings (community, hospitalization, and outpatient follow-up care). Their comprehensive recommendations were published in 2019 [25], providing much-needed guidance to support the delivery of high-quality AKI care through the establishment of clinical care pathways and guidelines. These pathways are designed to measure key quality indicators and to share strategies for progressive, systematic improvements of these metrics. At the heart of this discussion is an understanding of the principles of quality improvement

6

Division of Neonatology, Department of Pediatrics, School of Medicine and Public Health, University of Wisconsin-Madison, Madison, WI, USA

7 Division of Nephrology, Dialysis, and Transplantation, Stead Family Department of Pediatrics, University of Iowa, Iowa City, IA, USA

8 Department of Pediatrics, Division of Nephrology, Stanford University, Stanford, CA, USA

9 Department of Pediatrics, Toronto Hospital for Sick Children, Toronto, Canada

10 Department of Medicine, Department. Nephrology Dialysis \& Transplantation, International Renal Research Institute, San Bortolo Hospital, University of Padova, Vicenza, Italy

11 Center for Acute Care Nephrology, Cincinnati Children's Hospital Medical Center, Cincinnati, OH, USA

12 Department of Pediatrics, Texas Children's Hospital, Houston, TX, USA 
and their application to improving the care of patients with AKI laid out by the 22nd ADQI report (Fig. 1.)

The 22nd ADQI Conference was focused on AKI disease and the process of care in adults only. Although many of the approaches and processes of care recommendations provided by the 22nd ADQI consensus document may apply to pediatric AKI care, there are many unique aspects (including ascertainment, risk profiling, diagnostic approach, quality indicators, and strategies of care) that differ in children. Thus, pediatricspecific AKI pathways and some pediatric-specific quality metrics are needed to help improve AKI care in children.

To provide an interpretation of and framework for the pediatric community to apply the 22 nd ADQI Conference recommendations, a multidisciplinary group of pediatric AKI experts (nephrologists, nurse practitioners, neonatologists, and intensivists) convened by videoconference and by email on several occasions. The overarching goal of this activity was to assist the pediatric community in improving AKI care by providing pediatricspecific interpretations and guidance in the mobilization of the consensus statements put forth by the 22nd ADQI report.

This report reflects consensus expert opinions. For each domain, we first list the questions and statements from the 22nd ADQI report, then provide a summative, consensus pediatric opinion to each statement. This manuscript addresses the five areas highlighted by the 22nd ADQI report: (a) community health standards for AKI, (b) primary prevention of AKI during hospital encounters, (c) secondary prevention of AKI, (d) kidney support therapy quality indicators, and (e) tertiary prevention of short- and long-term AKI-related complications.

\section{Community healthcare standards for AKI}

\section{Question 1: At the community level, what are the roles and responsibilities of clinicians and healthcare systems in AKI risk monitoring and mitigation?}

22nd ADQI consensus statement A Healthcare systems and healthcare professionals should identify populations and patients at risk of $A K I$ and implement monitoring and preventive interventions to decrease the incidence of AKI.

22nd ADQI consensus statement B We suggest that a minimum set of baseline risk factors and acute exposures be considered for AKI risk stratification.

Pediatric patients can develop AKI in the community, on general care wards, or in the ICU. There are very little pediatric data on community-acquired AKI, yet a significant amount of pediatric AKI begins in the community. Data on the incidence of AKI in the non-ICU population are also limited, though several studies have demonstrated high rates of AKI in these patients associated with exposure to nephrotoxic medications [24, 26-28]. The Assessment of Worldwide AKI and Renal angina Epidemiology (AWARE) study, a multicenter study of over 5000 pediatric patients receiving care in intensive care units, showed that $15 \%$ of patients had AKI on the day of admission to the PICU [1]. The total rate of AKI at any point in the first 7 days was $27 \%$. These data suggest that most cases of AKI in the PICU occur before ICU admission [1] and highlight the principle that $\mathrm{AKI}$ awareness and prevention must start in the community and on non-ICU wards.

Developing strategies aimed at primary prevention before hospitalization may be critical to improving outcomes in pediatric AKI. Furthermore, because many pediatric patients who suffer AKI episodes have underlying chronic diseases, it is of paramount importance that strategies for primary prevention target not only outpatient pediatricians but also pediatric subspecialists and hospitalists. Pediatric populations at risk for AKI are outlined in Table $1[1-4,6,7,10,16-18$, 22, 27, 29-31]. Examples of procedures and disease states which are associated with AKI are outlined in Table 2 [1, 2, $10,11,18,24,30,32-35]$. Indeed, most pediatric patients in high-income countries with AKI do not have primary kidney disease $[1-6,16,22]$. Rather, most have a variety of comorbidities managed by primary care physicians and nonnephrology subspecialists in the ambulatory setting. Pediatric nephrologists should help educate primary care and
Fig. 1 Comprehensive quality improvement methodology. The seven steps that need to be taken for a successful quality improvement project (Define, Measure, Analyze, Implement, Control; PDSA, Plan, Do, Study, Act.) (25)

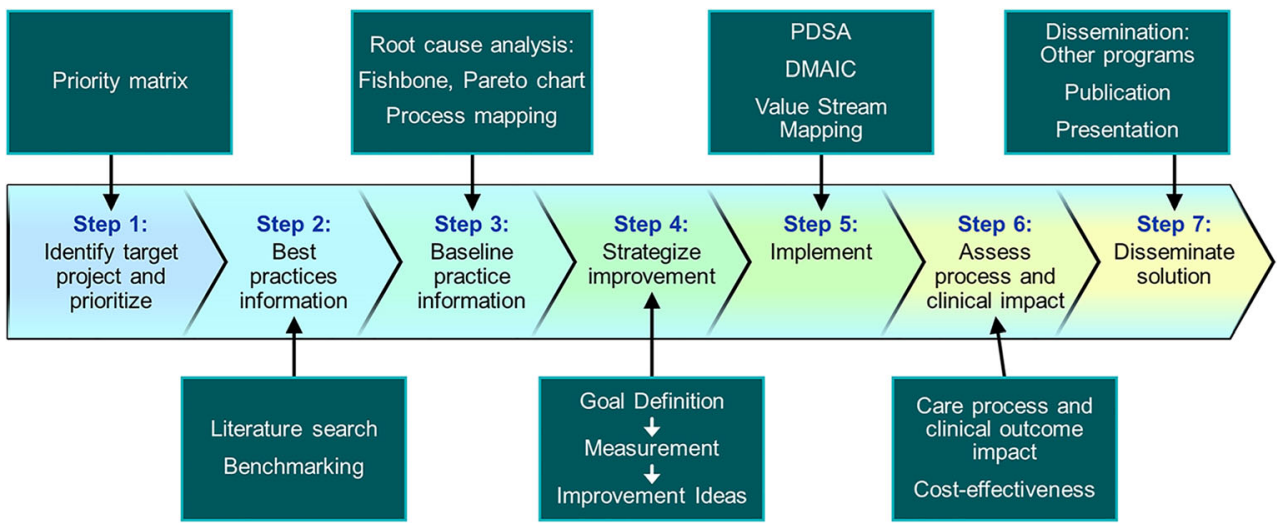


Table 1 Populations at risk for acute kidney injury

subspecialty pediatricians on the spectrum of issues related to AKI (prevention, recognition, follow-up, etc.).

The Global Snapshot, conducted by the International Society of Nephrology "0by25" AKI initiative, was a worldwide cross-sectional, observational study to evaluate AKI in hospitalized patients [36]. Of the pediatric patients with AKI, $53 \%$ of the children developed AKI while hospitalized and $47 \%$ in the community. Notably, the etiology of AKI in low- and middle-income countries was more likely to be due to dehydration, hypotension, infection, and primary kidney diseases. Therefore, strategies to prevent AKI at the community level may differ by region.

\section{Question 2: How should AKI high-risk populations be monitored?}

22nd ADQI consensus statement A We suggest populations/ patients at high risk for developing AKI should have a Kidney

Table 2 Examples of high-risk procedures and states

High-risk procedures
- Cardiopulmonary bypass
- ECMO
- Radiologic studies and procedures utilizing iodinated contrast
- Major surgical procedures
- Cancer treatment
High-risk states
- Dehydration
- Decreased oncotic pressure
- Hypotension
- High nephrotoxic medication exposure
- High-risk medical history as noted above
- Admission to intensive care unit
- Diarrheal illnesses
- High-risk infections (e.g., typhoid, cholera, dengue fever, malaria)

Health Assessment (KHA) at least every 12 months to define and modify their AKI risk profile.

22nd ADQI consensus statement B We suggest that high-risk patients have another KHA at least 30 days before AND again 2-3 days after a planned exposure that carries AKI risk. The KHA can be tailored to the clinical context and clinician/healthcare system judgment.

22nd ADQI consensus statement C We suggest that clinicians review a patient's KHA immediately after an unplanned acute exposure that carries AKI risk.

The 22nd ADQI report suggests that at-risk patients should have a KHA yearly and before or during an illness/procedure known to carry a high risk of AKI. The components of this assessment include the ABCDD, including (1) AKI history, (2) Blood pressure determination, (3) Creatinine measurement, (4) Drug list review, and (5) Dipstick of urine.

Although we agree in principle with performing a KHA, we suggest that the KHA be modified to a pediatric KHA (pKHA) such that it includes an AKI history, Blood pressure measurement according to the American Academy of Pediatrics (AAP) guidelines [37], serum Creatinine measurement and Drug list review (or $\mathrm{ABCD}$ evaluation; Fig. 2). The group believes that the urinalysis should not be mandatory in otherwise asymptomatic pediatric patients given concerns regarding high falsepositive rates in the pediatric population, particularly with regard to orthostatic proteinuria [38, 39]. In addition, many pediatric patients are incontinent and unable to provide a reliable cleancatch urine sample. Performance of a urinalysis may be considered by healthcare providers based on AKI or kidney history, as clinically indicated.

The group agrees that a pKHA assessment should be performed in patients at high-risk for AKI at least every 1-2 years, given the relatively low rates of chronic kidney disease (CKD) in children. This is less frequent than the recommendation of yearly evaluations in the $22 \mathrm{nd}$ ADQI report. The group agrees that the pKHA assessment should be undertaken before highrisk procedures, surgeries, and exposure to nephrotoxic medications. This assessment would then be utilized to help determine if appropriate preventive measures should be implemented (e.g., hydration before high-risk events). The group agrees with the recommendation that a repeat pKHA should occur 48-72 $\mathrm{h}$ after high-risk procedures, surgeries, exposures, and more often as clinically indicated.

\section{Question 3: How can AKI preventive strategies be implemented within high-risk populations?}

22nd ADQI consensus statement A KHA should be followed by a kidney health response (KHR) after acute exposure to risk factors of $A K I$. 
Fig. 2 Pediatric Kidney Health Assessment and Response

\section{Population}

(National monitoring for variation in AKI incidence)

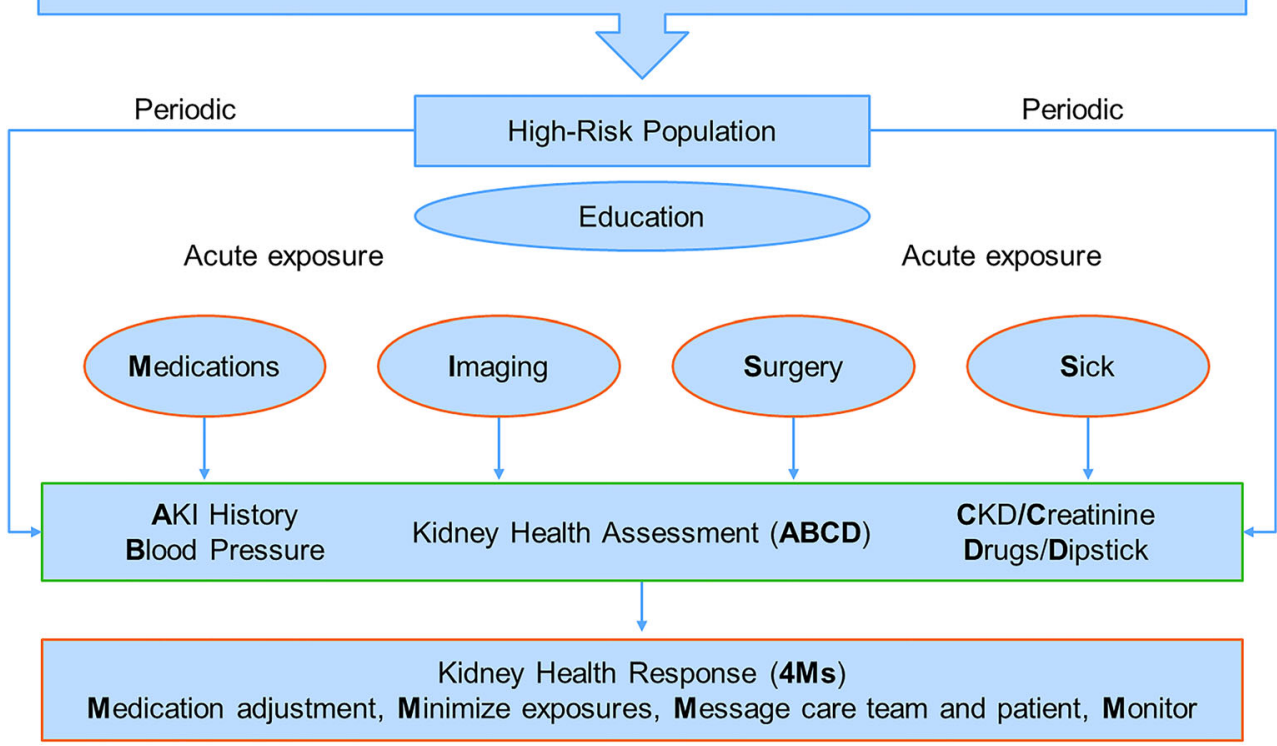

22nd ADQI consensus statement B We suggest raising awareness of the definition, signs, symptoms, and acute exposures associated with AKI among clinicians and high-risk patients/populations.

22nd ADQI consensus statement C We suggest enhanced coordination between all stakeholders to monitor the rate, causes, and outcomes of AKI to identify variations in care and outcomes across and between the populations.

The expert panel strongly agrees with these strategies to prevent AKI and feel that they are broadly applicable to pediatrics. The 22nd ADQI report consensus statement underscores the fact that the KHA is a snapshot of the current state of kidney health, and assessment and evaluation of the changes over time are invaluable to making an appropriate "kidney health response." The KHR focuses on "the 4Ms": (1) Medication adjustment, (2) Minimize exposures, (3) Message care team and patient, and (4) Monitor.

The 22nd ADQI report highlights the importance of coordination between healthcare staff to mitigate AKI risk in high-risk populations. Coordination of care is most essential when there are potential benefits and risks to interventions. This recommendation is particularly applicable to the increasingly complex pediatric patients comanaged by multiple subspecialists at tertiary care centers. Examples of situations where coordination may be essential include implementation of protocols designed to prevent tumor lysis-associated AKI in pediatric oncology patients [40, 41], balancing risks and benefits of contrast administration during cardiac procedures in children with congenital heart disease, and choice of antibiotics in patients exposed to multiple nephrotoxic medications. We recognize that these decisions are often complex and require input from multidisciplinary care team members. At a minimum, communication and strategies to help identify high-risk patients and mitigate AKI when possible likely reduce the incidence of AKI. Table 1 provides examples of populations that may benefit from such protocols.

In the absence of proven treatments for established AKI, preventive strategies are the foundation for AKI care in children. Coordinated multidisciplinary efforts to build awareness and to educate the primary care and subspecialty pediatric community on issues related to pediatric AKI (definition, signs/symptoms, high-risk exposures, high-risk populations, prevention, recognition, follow-up, etc.) are greatly needed. We propose that the pediatric nephrology community should actively lead this effort.

The involvement and engagement of general pediatricians, pediatric hospitalists, non-nephrology subspecialists, and pediatric intensivists will be central to efforts designed to decrease the burden of AKI. Furthermore, on an individual level, pediatric nephrologists should make themselves available for consultation before high-risk procedures in high-risk populations to optimize pre-exposure care. Community primary and subspecialty clinical care providers who care for children at risk for AKI should have access to appropriate blood pressure monitoring systems that fit all sized patients and have the ability to obtain urine and blood testing. 


\section{Primary prevention of AKI during a hospital encounter}

\section{Question 1: How and when should the risk for AKI be identified among hospitalized patients?}

22nd ADQI consensus statement A All patients at hospital admission should be screened for the risk of AKI. Screening should occur at the earliest possible time throughout the patient's hospital stay.

22nd ADQI consensus statement B All patients at risk for AKI should at least have an assessment of serum creatinine, urine dipstick analysis, and urine output measurement. Complementary diagnostic tests depending on local availabilities, risk factors, clinical context, and clinician judgment should be considered.

22nd ADQI consensus statement C All hospitalized patients should have periodic risk reassessment using appropriate clinical or electronic models before and after risk exposure or change in clinical status.

Once children are admitted to the hospital, there should be a transition in AKI management to early recognition and the implementation of mitigation strategies to improve outcomes. In hospitalized children, the timely recognition of AKI and the early implementation of AKI mitigation strategies are critical to improving outcomes. The expert panel agrees with the 22nd ADQI statement that during each pediatric hospital admission, a child should undergo an assessment to determine risk factors for AKI development during that admission. The appropriate timing of risk assessments depends on the environment but can occur in the emergency department, pre-operative surgical area, and near or around the time of transfer to the ICU. The risk factors inherent to comorbid disease and the admitting diagnosis are outlined in
Tables 1 and 2. These include but are not limited to intravascular volume depletion, nephrotoxic medication exposure, hypotension, sepsis or suspected infection, oncologic disease, specific perioperative patients, vasoactive support, a history of transplantation requiring immunosuppression, and receipt of extracorporeal membrane oxygenation (ECMO). Scoring systems can be devised in a simple, pragmatic manner, specific to the healthcare context, to stratify patients by level of risk. An example of a risk methodology is the renal angina prodrome - operationalized in the pediatric intensive care unit, whereby the identification of risk factors at the time of ICU admission, combined with a few biological measures in the first $12 \mathrm{~h}$ of ICU admission (Table 3), are used to predict who will develop severe AKI at $72 \mathrm{~h}$ of ICU admission. Derived initially in a multicenter PICU study and validated in the Assessment of Worldwide AKI, Renal Angina, and Epidemiology (AWARE) study, the renal angina index (RAI) demonstrates a higher negative predictive value for no AKI and higher specificity for severe AKI than isolated markers of kidney function without context (Table 3) [29, 42, 43]. The electronic medical record (EMR) can be utilized to provide a RAI score at ICU admission to risk-stratify patients. Similarly, in hospitalized children at high-risk for nephrotoxin-associated $\mathrm{AKI}$, the EMR has been leveraged to identify patients at risk for AKI in both the general care ward and ICU settings [26-28]. The development of risk stratification systems mobilizing the EMR across a variety of settings in pediatric hospitals (emergency department, perioperative cardiac intensive care, general pediatric wards, etc.) and the incorporation of these into the EMR should be a future priority.

If the assessment suggests high risk for AKI, we agree with the 22nd ADQI report that a serum creatinine should be measured and urine output monitored. Although the 22nd ADQI report recommended a urinalysis in adult patients at high AKI risk, the expert panel did not firmly recommend urinalysis for all pediatric patients due to the concerns about high false-positive rates previously mentioned. A urinalysis should be considered in

Table 3 Renal angina index [42]

\begin{tabular}{|c|c|c|c|}
\hline \multicolumn{4}{|l|}{ Risk level } \\
\hline Patient type & Risk level & Score & \multirow{6}{*}{ Renal angina rules in at $\geq 8$} \\
\hline Sepsis or ICU admission & Moderate & 1 & \\
\hline Diabetes or stem cell transplant & High & 3 & \\
\hline Ventilation or ionotropy & Very high & 5 & \\
\hline \multicolumn{3}{|c|}{$\mathbf{X}$} & \\
\hline & Injury level & & \\
\hline $\mathrm{SCr} /$ baseline $\mathrm{Cr}$ & Percent fluid overload & Score & \\
\hline Decreased or no change & $<5 \%$ & 1 & \\
\hline$>1-1.49$ times & $5-10 \%$ & 2 & \\
\hline$>1.5-1.99$ times & $10-15 \%$ & 4 & \\
\hline$\geq 2$ times & $>15 \%$ & 8 & \\
\hline
\end{tabular}


the appropriate clinical scenario where it is likely to provide actionable data and/or assist with differential diagnosis. The 22nd ADQI report did not comment on trending of fluid balance. Based on the mounting evidence surrounding the negative impact of fluid overload in critically ill patients, we feel strongly that all pediatric patients at risk for AKI should have a careful assessment of their current fluid status. A fluid evaluation can be done using signs and symptoms of intravascular volume depletion/ fluid overload and can be enhanced with careful evaluation of daily fluid balance and weights during the hospitalization. Evaluation of cumulative fluid balance (as opposed to only reporting the fluid balance for the past $24 \mathrm{~h}$ ) should be part of the daily assessment in critically ill children at risk for AKI.

The group also felt strongly that pediatric AKI assessments in high-risk groups should be performed at admission and daily, while the patient remains at risk for AKI. Reassessments of changes in serum creatinine, urine output, and fluid balance should be done daily and more often if necessary. The frequency of assessments should be tailored to the individual patient and disease, with repetition as a patient's condition changes and risk escalates.

Just as in the identification of patients at high risk for AKI, the EMR can also be leveraged for the timely diagnosis of AKI when it occurs. Some centers have incorporated an EMR alert for the presence of AKI in hospitalized patients that then provides recommendations on management (e.g., in the form of a care bundle) as outlined in this document. The goals of these alert systems are not only to enhance provider awareness but also to reduce AKI severity and duration. However, the benefits of these AKI alert tools remain to be determined $[44,45]$.

\section{Question 2: What core preventive measures should be considered as a target for quality improvement projects?}

22nd ADQI consensus statement A Early correction or mitigation of context-specific modifiable risk factors of AKI should be considered for all high-risk patients.

A pediatric-specific AKI care bundle should be developed and used to correct or mitigate risk factors in children across the hospital spectrum. Such a bundle would be centered around the identification of AKI, mitigation of risk factors, and monitoring for preventable complications (Table 4). We recognize that different strategies and bundles may be required for different patient populations (i.e., cardiac vs. oncology) and clinical scenarios. Working with subspecialty teams who understand and can balance the pros and cons of interventions in the context of holistic patient care will be essential to the implementation of such bundles.

The pediatric consensus group identified core measures including optimizing hemodynamics, reducing the number of avoidable nephrotoxic exposures, placement of an indwelling
Table 4 An example of the components which may be found in a pediatric AKI bundle

Evaluation of potentially modifiable risk and complications of AKI

- Cardiopulmonary bypass

- Monitor intake/output, weights, kidney function panel, cumulative volume status at least daily

- Evaluate medications for nephrotoxic potential

- Evaluate and treat the underlying AKI cause

- Hypotension/low cardiac output/ low renal perfusion

- Low oncotic pressure/low serum albumin

- Bladder obstruction

- Evaluation of nutrition/electrolyte composition of fluid intakes

- Multidisciplinary approach: pharmacy, dietitian, bedside nurses

- Notify nephrology for progressive kidney dysfunction, severe electrolyte problems, or progressive fluid overload

bladder catheter to monitor urine output as indicated, and optimization of intravascular oncotic pressure. Furthermore, we would advocate that serial measurement of volume status (ideally daily weights, but fluid balance at a minimum), and tracking of cumulative fluid balance should be a mandatory part of the daily evaluation in children with and at high-risk for AKI [46, 47]. Finally, the group believes the expertise of multidisciplinary team members, including clinical pharmacists, dietitians, and nurses, is a critical component to mitigate the consequences of AKI.

\section{Question 3: What are the quality indicators for assessing risk of AKI?}

22nd ADQI consensus statement A The quality indicators for AKI prevention in the hospital should include (1) proportion of patients screened for AKI risk among all admissions, (2) proportion of identified AKI high-risk patients among all screened patients, (3) proportion of AKI high-risk exposures (e.g., medication, contrast, surgery) among all hospitalized population and all high-risk patients, (4) proportion of patients who received an appropriate intervention around a high-risk exposure, and (5) proportion of patients who eveloped AKI among all admissions (in different subpopulations) and all high-risk patients.

22nd ADQI consensus statement B The quality indicators should be reviewed and utilized to identify areas of improvement and action. The frequency of reporting should be defined according to local resources and regulatory requirements.

The expert panel feels that these statements are broadly applicable to pediatric patients and strongly encourage their incorporation into programs. In fact, many of these quality improvement metrics were first developed and used in pediatric programs. Pediatric providers have been leaders in the development of quality improvement projects to reduce harm 
from nephrotoxic medications. The Nephrotoxic Injury Negated by Just-in-time Action (NINJA) project utilizes the EMR to identify patients exposed to a nephrotoxic medication burden and trigger serum creatinine surveillance. Multiple studies have clearly shown that this results in sustainably decreased rates of AKI, decreased costs, and improved outcomes [26-28, 48]. Today, there are over 40 pediatric hospitals that are using these metrics to track quality improvement efforts to reduce hospital-acquired nephrotoxic medication associated with AKI as part of the Solutions for Patient Safety collaborative. The NINJA project represents a prime example of how the EMR can be mobilized to identify patients at high-risk of AKI. An example of a written notification provided utilizing the NINJA mechanism is provided as Supplemental Figure 1. These quality improvement metrics for nephrotoxic medication-associated AKI can be applied to other cohorts and could serve to develop an institutional AKI dashboard.

Multicenter pediatric studies have demonstrated that there are significant differences in AKI epidemiology across institutions $[1,10]$. It is essential to understand the baseline rates of these metrics at the institutional level before implementing interventions designed to improve outcomes. The stated metrics can be utilized to monitor practices, determine the effectiveness of interventions, identify benchmarks for care, and develop institutional quality improvement goals centered around AKI reduction.

Further development, validation, and use of such metrics utilizing the comprehensive quality improvement methodology outlined in Fig. 1 incorporating the tools provided by the EMR are needed to help identify opportunities for care improvement. Such an endeavor could take the form of an institutional AKI "dashboard" with the following potential metrics: (1) proportion of admitted patients evaluated to determine their risk for AKI during hospitalization, (2) proportion of screened patients identified as high risk, (3) proportion of high-risk exposures in high-risk patients, (4) number of atrisk patients who develop AKI, (5) AKI duration (days with AKI) in those who develop AKI, (6) incidence of AKI after $24 \mathrm{~h}$ of admission, (7) incidence of nephrotoxic-associated AKI, and (8) progression of AKI from hospital admission.

\section{Secondary prevention of AKI}

\section{Question 1: What are the key considerations for developing quality programs that evaluate contributors to an episode of AKI?}

22nd ADQI consensus statement A We propose that for each patient diagnosed with AKI during hospitalization, the goal is recovery to baseline kidney function in the shortest period of time with minimum number of complications. This is best achieved by timely and accurate diagnosis and management of AKI and prevention of complications. These goals might be achieved by using a Recognition-Action-Results framework.

22nd ADQI consensus statement B Quality improvement surrounding the diagnostic evaluation of AKI should attempt to maximize the proportion of patients who undergo a context-appropriate and timely evaluation while avoiding unnecessary testing.

We agree with the statements as they relate to the secondary prevention of pediatric AKI. The secondary prevention of AKI encompasses a two-pronged approach aimed at the mitigation of AKI severity and the prevention of AKI complications. At the heart of quality improvement strategies is a deep understanding of the contextual setting of AKI. Secondary preventive measures that are designed to halt AKI progression include strategies designed to alleviate potentially reversible causes of AKI, such as hypovolemia, low oncotic pressure, urinary obstruction, and intra-abdominal hypertension. Strategies designed to reduce AKI during high-risk events (e.g., limiting nephrotoxic medications in patients with AKI, optimization of hemodynamics) should also be implemented. After the timely diagnosis of AKI, quality improvement measures aimed at reducing the severity and duration of AKI episodes should focus on potentially modifiable factors and limiting consequences (e.g., hyperkalemia, fluid overload, acidosis).

The development, implementation, and dissemination of a "Pediatric Preventive AKI Bundle" is a meaningful goal for the pediatric critical care nephrology community. The efforts to develop context-specific AKI bundles have been successful in adults [49-54], but data is just now becoming available in the pediatric community [55]. These bundles would focus on education, risk assessment, timely diagnosis, interventions, mitigating consequences, and therapeutic monitoring. Leveraging the EMR may allow clinicians timely recognition of AKI and evaluation of critical metrics.

Evaluation of context-specific tests should start with understanding the pre-test probability of AKI based on risk factors at presentation, such that the patients who are most likely to benefit from screening will be evaluated. The specific risk factors and tests for different scenarios represent opportunities for quality improvement utilizing the methodology outlined in Fig. 1.

\section{Question 2: What are the key considerations for developing quality improvement programs focused on limiting the duration and severity of AKI?}

22nd ADQI consensus statement A Quality improvement programs should include the implementation and reporting of the proportion of patients that receive timely and diagnosis-appropriate interventions. Adherence with the locally agreed upon preventive interventions should be audited and shared with clinicians periodically. 
We agree that quality improvement programs should not only establish the process for care (which patients and which tests) but should also capture consistency in the application of standardized care processes. These include measures of the frequency of AKI assessment (e.g., percent of high-risk patients for whom a serum creatinine is measured), timely diagnosis, and appropriate interventions of strategies designed to reduce AKI. The dissemination of the data to key stakeholders is essential for quality improvement.

\section{Question 3: What are the key considerations for developing quality improvement programs focused on reducing the complications of AKI?}

22nd ADQI consensus statement A Quality indicators for prevention of avoidable AKI-related complications include monitoring, reporting the context-specific adverse events (to patient advocates, clinicians, administrators, and regulatory bodies), and implementation of risk reduction strategies.

Emerging data suggest that the consequences of AKI, such as fluid overload, electrolyte, and acid/base perturbations, and hypertension, are common and associated with poor outcomes in pediatric and adult cohorts [46, 47, 56-58]. There have been multiple pediatric studies demonstrating the negative impact of fluid overload and AKI [46, 47, 58-60]. Quality improvement programs designed to limit pediatric fluid overload in the context of a patient who is at risk for AKI should be prioritized. The new pediatric sepsis guidelines recommend early use of vasopressors and limiting "maintenance" fluids in pediatric patients with septic shock [61-64]. Strategies to reduce other consequences of AKI should be studied as they may lead to an improvement in outcomes.

\section{Kidney replacement therapy quality indicators}

\section{Question 1: How should the quality of acute kidney replacement therapy be monitored, evaluated, and reported?}

22nd ADQI consensus statement A Quality indicators should integrate structure, process, and outcome indicators for each therapeutic modality.

The pediatric expert panel agrees with the 22nd ADQI report that the systematic incorporation of quality indicators into acute kidney care programs is an essential part of all programs. The paradigm of kidney replacement therapy (KRT) in pediatrics has evolved, shifting from being a heroic therapy to standard of care for moderate to severe AKI. Kidney replacement therapies include peritoneal dialysis (PD), hemodialysis (HD), slow continuous ultrafiltration (SCUF), and continuous kidney replacement therapy (CKRT). Patient and provider characteristics, as well as institutional resources, determine which therapy is selected. While there remains controversy regarding the modality and timing of therapy, there is now a consensus within the community that programs should use quality improvement methodologies to ensure safe and high-quality care [25].

Pediatric CKRT (previously termed continuous renal replacement therapy) should be viewed as any other complex, high-risk procedure. Pediatric programs must have outlined policies and procedures, clearly defined roles and responsibilities for all team members, assure adequate training, develop procedure checklists, utilize timeouts for initiations (Supplemental Figure 2), and perform continuous quality improvement assessments. All programs should integrate structural, process, and outcome measures within the context of a quality improvement framework. Programs should focus on the most important elements that will capture the strengths and weaknesses within the program. These may include processes to optimize the initiation of therapy, assess the efficiency of therapy, evaluate provider skills, and evaluate patient safety [65]. As with any procedure in critical care, quality improvement in CKRT should seek to optimize the timing of the initiation, evaluate safety events, and optimize the efficiency of therapy.

\section{Question 2: What are the minimum structure quality indicators that should be implemented for acute KRT?}

22nd ADQI consensus statement A Structural quality indicators should specifically target clinician, nursing, and allied health professionals' capacity and expertise for providing acute KRT and identify a responsible team to implement and report quality metrics for acute KRT services.

Developing a sound infrastructure for an acute KRT program is the foundation for providing seamless, high-quality care across all modalities of acute KRT. The pediatric expert panel agrees that all pediatric acute KRT programs must have a set of physician/nursing champions with allocated administrative time to enable them to develop and maintain the programs [65], which at a minimum, includes the development and periodic update of procedures, policies, educational curricula, quality indicators, data collection, data analysis, and program evaluation $[65,66]$. This group must also incorporate a team of multidisciplinary experts with specific skills and expertise in the pediatric environment. The team make-up should include physicians, advanced practice providers (e.g., nurse practitioners/physician assistants), clinical nurse specialists, bedside nurses, a dietitian, and a clinical pharmacist with training in pharmacokinetics, pharmacodynamics, and medication dosing in patients with kidney failure and on KRT. The pharmacist also serves a critical role in pharmacyrelated solution-related logistic issues (e.g., dialysis solutions and anticoagulation). Furthermore, the delivery of KRT for 
critically ill pediatric patients requires a partnership between nephrologists and intensivists due to the complexity of the procedure, disease processes, and goals of care. Measures that evaluate the effectiveness of the team structure and its function should be implemented.

Each program must develop and maintain competent and well-trained providers who can provide safe and effective care. Physicians and advanced practice providers should have specific education and experience in prescribing acute KRT, while nursing personnel should have training in the skills and knowledge necessary for KRT delivery. Programs should establish minimum education and competency requirements which incorporate minimum standards and specific measures for monitoring the educational curriculum. All staff should have skills and knowledge assessed and reinforced over time. Programs with low procedure frequency should consider other educational opportunities such as modules and simulationbased education to develop and maintain the skill set specific to care delivery.

\section{Question 3: What are the minimum process quality indicators that should be implemented for the provision of acute KRT?}

22nd ADQI consensus statement A Process quality indicators should incorporate methodologies that lead to standardized protocols and procedures, allowing for increased efficiency and consistency in care and safety, and should be specific to each KRT modality.

The pediatric expert panel agrees with the importance of process indicators, which are essential to the safe delivery of care. This is especially true in pediatric patients, given the challenges posed by the breadth of patient sizes. The standardization and elimination of practice variation should be developed with key stakeholders using current evidence and identified best practices. The process of protocol development and maintenance should be iterative in nature with regularly scheduled evaluations of established practices. Agreed upon policies, protocols, and procedures should guide practices along the continuum of KRT care from consultation to discontinuation of therapy (e.g., timing, prescribing, initiation, vascular access insertion/care, dialysis dosing, anticoagulation, medication modifications, nutrition).

Process quality measures should focus on adherence to institutional standard practice guidelines, such as placement/ maintenance of vascular access, timing of therapy, prescription, anticoagulation, nutrition, filter life, and medication management. Appropriate vascular access is critical to providing adequate acute kidney support therapy [67]. Weight-based guidelines should be utilized to recommend the size and location of vascular access [68]. In institutions with interventional radiology or pediatric surgical teams, a cuffed, tunneled catheter should be considered if the duration of therapy is expected to be greater than 2-3 weeks in the absence of a contraindication (e.g., infection) [68]. We recommend that quality improvement initiatives assess adequate delivery of therapy, including vascular access measures (i.e., proportion of catheters/treatment, number of catheter revisions, CLABSI, adherence to placement guidelines).

We also recommend that quality initiatives assess filter life. Filter life assessment provides insights into the process of care and understanding the reasons for treatment interruptions. Programs should measure filter life (time from the filter start to filter end) and categorize the reason for filter discontinuation. The two classifications commonly utilized as quality measures to describe filter discontinuation are "planned" (filter hours that are greater than or equal to the institutional standard) and "unplanned" (filters discontinued before the institutional standard). Filter changes due to changing patient conditions or procedures that require disconnection from the circuit are labeled as planned. Unplanned filter changes (e.g., clotted filters) provide insight into potential programmatic issues and evaluation/root cause analysis may identify opportunities (educational, protocol modifications) to optimize therapy delivery [66].

The optimal timing for the initiation of acute KRT remains an active area of research. We recommend that quality initiatives should include measurement of fluid overload status and AKI staging at the time of therapy initiation [47, 58]. The initiation of acute KRT should be done according to the established practices associated with all critical procedures. These practices include performing a "time-out" using a checklist to review the prescribed procedure, CKRT prescription, vital sign goals, and the first and second treatments for vital sign changes. Additionally, at each pediatric CKRT initiation procedure, a provider (physician, physician assistant, or nurse practitioner from critical care and nephrology) should be present due to the dynamic and complex patient conditions. Supplemental Figure 2 provides such an example of a time-out outlining the personnel, roles, responsibilities, and process surrounding the procedure of CKRT initiation.

\section{Question 4: What are the minimum outcome indicators that should be implemented for the provision of acute KRT?}

22nd ADQI consensus statement A Outcome quality indicators should include patient-centered outcomes, including clinician and patient satisfaction, mortality, and quality of life among survivors; dialysis liberation rates; and health-economic outcomes.

Institutions should track metrics that evaluate outcome measures. Pediatric-specific metrics should include parental understanding, parental satisfaction, and pediatric-specific quality of life. 


\section{Tertiary prevention of AKI short- and long-term complications}

\section{Question 1: How should the appropriate post- AKI/acute kidney disease care be measured?}

\section{2nd ADQI consensus statement A Healthcare systems need to quantitate the proportion of patients who need post-AKI/acute kidney disease (AKD) follow-up, as well as those who receive any post-AKI/AKD follow-up, and evaluate quality of care for those who received post-AKI/AKD follow-up.}

Programs should seek to optimize follow-up as the final step in the care of pediatric patients following an episode of AKI. Efforts should be made to ensure that follow-up visits are scheduled prior to discharge. The communication of clinical information to outpatients is a critical component to the follow-up of children with AKI. At the most basic level, problem lists should be updated and reviewed for accuracy with the specific inclusion of episodes of AKI and risk factors for AKI. This ensures providers can recognize those at risk for future episodes of AKI, especially those who have had multiple episodes of AKI. Follow-up care may require the development of specific AKI follow-up clinics at busier pediatric institutions. At a minimum, the following groups warrant consideration for follow-up by pediatric nephrologists: those with a history of severe AKI (stage 2, stage 3, or kidney support therapy), persistent AKI (AKI lasting $\geq 3$ days $[69,70]$ ), cohorts with other risk factors for CKD (e.g., congenital heart disease, solid organ transplants, stem cell transplants, oncologic disease, severe prematurity), and patients who have suffered multiple episodes of AKI.

\section{Question 2: What are the key elements of an appropriate post-AKI/AKD care bundle?}

22nd ADQI consensus statement A Quality indicators should at least include structure (needed personnel and resources), process for follow-up (who and by whom, what, where, when, why, and how), and outcome indicators (CKD progression, continued or new need for dialysis, mortality, etc.).

'To ensure adequate follow-up, acute kidney support programs should have the initial follow-up performed by a pediatric nephrologist within 3 months of the discharge, as recommended by the KDIGO guidelines [68]. This follow-up should include kidney function and blood pressure measurements. Additional testing such as urine dipstick, urinalysis, or kidney ultrasound can be considered on a case-by-case basis. Patients and their families should be educated about AKI and their subsequent risk. (Supplemental Figure 3 presents an example of supplemental patient educational material.) The duration of subsequent follow-up should be determined based on the presence of and risk for subsequent CKD, availability of local resources, and willingness for primary care physicians to follow patients at risk for CKD. There remains a glaring need for research studies to assess long-term AKI outcomes and to determine the optimal timing, duration, and process for AKI follow-up across different healthcare contexts. Programs should track the rates of follow-up
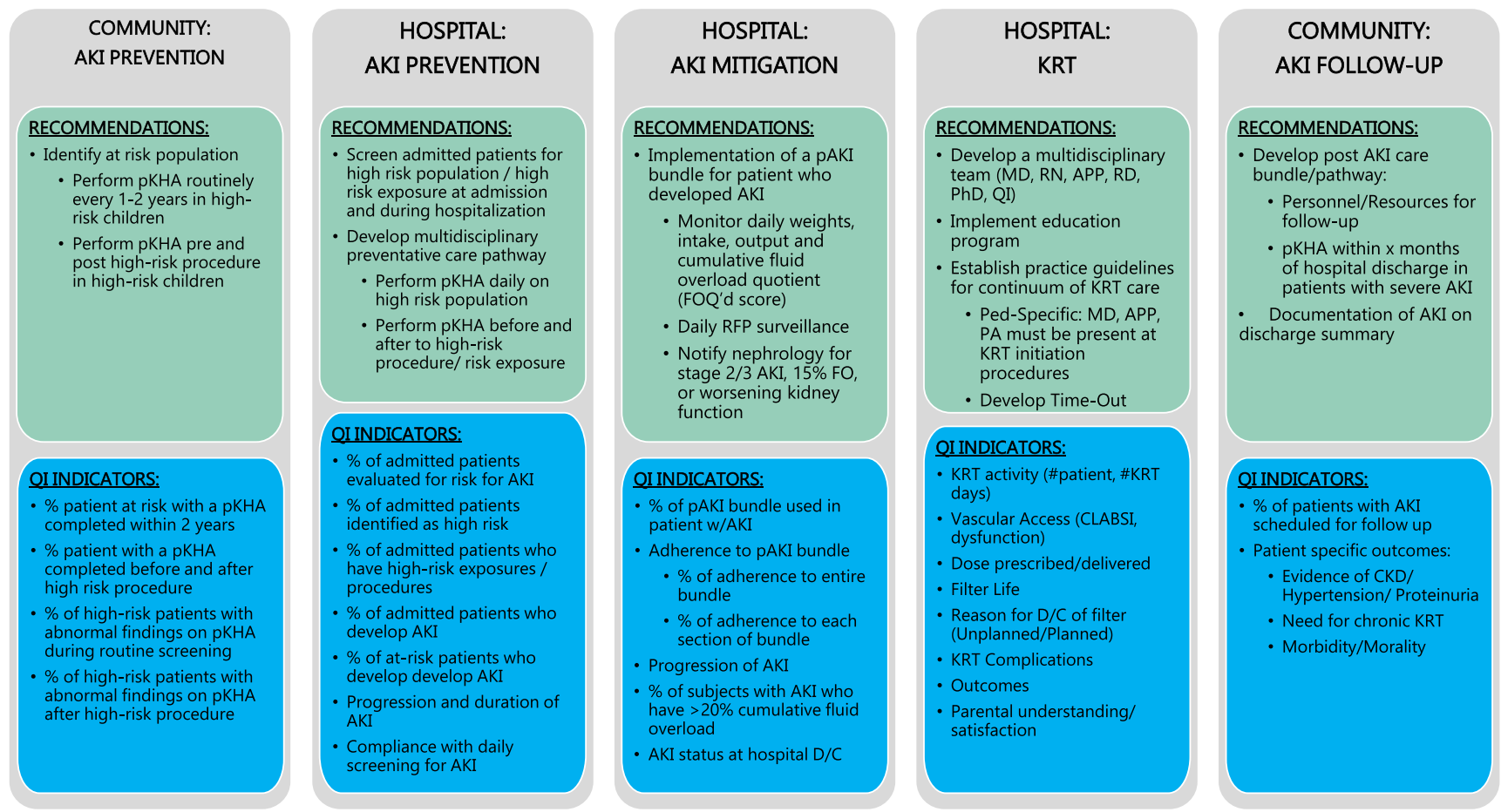

Fig. 3 Pathway for the application of the 22nd ADQI Consensus Statements to pediatric clinical care 
and the accuracy of problem lists as quality indicators. As programs increasingly incorporate telemedicine, it will be critical to consider and research technologies (remote laboratory testing and blood pressure measurement) and strategies to optimize the follow-up of children with AKI.

\section{Summary statement}

In summary, the authors of this interpretation of the 22nd ADQI Consensus Statements have attempted to provide a perspective and pathway for its application to pediatric clinical care, summarized in Fig. 3. As the experts in the field of pediatric critical care nephrology, we strongly recommend consideration of each unique aspect of the 22nd ADQI report. Beyond this, we strongly suggest the need for a formalized review of the available evidence specific to pediatric epidemiology, diagnostics, extracorporeal therapies, and kidney support, bio-pathophysiology, and education to provide the basis for dedicated quality improvement initiatives in children. Quality improvement offers tremendous opportunities for research, education, and practice improvement for pediatric patients at risk for AKI and its sequelae.

Supplementary Information The online version contains supplementary material available at (https://doi.org/10.1007/s00467-020-04828-5).

Acknowledgments We acknowledge Drs. Ravi Mehta, John Kellum, and Claudio Ronco for their leadership and vision in leading the ADQI collaborative. Furthermore, we are indebted to all of the members of the 22nd ADQI meeting who worked together to develop and articulate the importance of quality improvement in the field of AKI.

\section{Compliance with ethical standards}

Conflict of interest The authors have no quality improvement-related conflicts of interest to report that would have affected the writing of this publication. For AKI-related research, Michael Zappitelli is a member of the AKI adjudication committee for an industry-sponsored study on NGAL as an AKI biomarker (Bioporto Inc.). David Selewski has participated in AKI adjudication for an industry-sponsored study on NGAL as an AKI biomarker (Bioporto Inc.). David J Askenazi is consultant for Baxter, CHF solutions, Medtronic, AKI Foundation, and Bioporto. Rajit Basu is a consultant for Baxter, Biomerieux, and BioPorto. Theresa Mottes is consultant for Baxter and Medtronic. Claudio Ronco consulted or participated in advisory boards for ASTUTE, ASAHI, Baxter, Biomerieux, OCD, GE, FMC, Cytosorbents, B. Braun, Medtronic, Jafron, and Toray over the last 3 years.

\section{References}

1. Kaddourah A, Basu RK, Bagshaw SM, Goldstein SL, AWARE Investigators (2017) Epidemiology of acute kidney injury in critically ill children and young adults. N Engl J Med 376:11-20

2. Fleming GM, Sahay R, Zappitelli M, King E, Askenazi DJ, Bridges BC, Paden ML, Selewski DT, Cooper DS (2016) The incidence of acute kidney injury and its effect on neonatal and pediatric extracorporeal membrane oxygenation outcomes: a multicenter report from the kidney intervention during extracorporeal membrane oxygenation study group. Pediatr Crit Care Med 17:1157-1169

3. Sutherland SM, Byrnes JJ, Kothari M, Longhurst CA, Dutta S, Garcia P, Goldstein SL (2015) AKI in hospitalized children: comparing the pRIFLE, AKIN, and KDIGO definitions. Clin J Am Soc Nephrol 10:554-561

4. Selewski DT, Cornell TT, Heung M, Troost JP, Ehrmann BJ, Lombel RM, Blatt NB, Luckritz K, Hieber S, Gajarski R, Kershaw DB, Shanley TP, Gipson DS (2014) Validation of the KDIGO acute kidney injury criteria in a pediatric critical care population. Intensive Care Med 40:1481-1488

5. Akcan-Arikan A, Zappitelli M, Loftis LL, Washburn KK, Jefferson LS, Goldstein SL (2007) Modified RIFLE criteria in critically ill children with acute kidney injury. Kidney Int 71:1028-1035

6. Alkandari O, Eddington KA, Hyder A, Gauvin F, Ducruet T, Gottesman R, Phan V, Zappitelli M (2011) Acute kidney injury is an independent risk factor for pediatric intensive care unit mortality, longer length of stay and prolonged mechanical ventilation in critically ill children: a two-center retrospective cohort study. Crit Care 15:R146

7. Fitzgerald JC, Basu RK, Akcan-Arikan A, Izquierdo LM, Pineres Olave BE, Hassinger AB, Szczepanska M, Deep A, Williams D, Sapru A, Roy JA, Nadkarni VM, Thomas NJ, Weiss SL, Furth S, Sepsis PRevalence, OUtcomes, and Therapies Study Investigators and Pediatric Acute Lung Injury and Sepsis Investigators Network (2016) Acute kidney injury in pediatric severe sepsis: an independent risk factor for death and new disability. Crit Care Med 44: 2241-2250

8. Fitzgerald JC, Ross ME, Thomas NJ, Weiss SL, Balamuth F, Anderson AH (2018) Risk factors and inpatient outcomes associated with acute kidney injury at pediatric severe sepsis presentation. Pediatr Nephrol 33:1781-1790

9. Garcia RU, Natarajan G, Walters HL, Delius RE, Aggarwal S (2018) Acute kidney injury following first-stage palliation in hypoplastic left heart syndrome: hybrid versus Norwood palliation. Cardiol Young 28:261-268

10. Blinder JJ, Asaro LA, Wypij D, Selewski DT, Agus MSD, Gaies M, Ferguson MA (2017) Acute kidney injury after pediatric cardiac surgery: a secondary analysis of the safe pediatric euglycemia after cardiac surgery trial. Pediatr Crit Care Med 18:638-646

11. Blinder JJ, Goldstein SL, Lee VV, Baycroft A, Fraser CD, Nelson D, Jefferies JL (2012) Congenital heart surgery in infants: effects of acute kidney injury on outcomes. J Thorac Cardiovasc Surg 143: 368-374

12. McGregor TL, Jones DP, Wang L, Danciu I, Bridges BC, Fleming GM, Shirey-Rice J, Chen L, Byrne DW, Van Driest SL (2016) Acute kidney injury incidence in noncritically ill hospitalized children, adolescents, and young adults: a retrospective observational study. Am J Kidney Dis 67:384-390

13. Menon S, Kirkendall ES, Nguyen H, Goldstein SL (2014) Acute kidney injury associated with high nephrotoxic medication exposure leads to chronic kidney disease after 6 months. J Pediatr 165: 522-527 e522

14. Misurac JM, Knoderer CA, Leiser JD, Nailescu C, Wilson AC, Andreoli SP (2013) Nonsteroidal anti-inflammatory drugs are an important cause of acute kidney injury in children. J Pediatr 162: 1153-1159 1159 e1151

15. Moffett BS, Goldstein SL (2011) Acute kidney injury and increasing nephrotoxic-medication exposure in noncritically-ill children. Clin J Am Soc Nephrol 6:856-863

16. Schneider J, Khemani R, Grushkin C, Bart R (2010) Serum creatinine as stratified in the RIFLE score for acute kidney injury is associated with mortality and length of stay for children in the pediatric intensive care unit. Crit Care Med 38:933-939 
17. Cornell TT, Selewski DT, Alten JA, Askenazi D, Fitzgerald JC, Topjian A, Holubkov R, Page K, Slomine BS, Christensen JR, Dean JM, Moler FW (2018) Acute kidney injury after out of hospital pediatric cardiac arrest. Resuscitation 131:63-68

18. Rheault MN, Zhang L, Selewski DT, Kallash M, Tran CL, Seamon M, Katsoufis C, Ashoor I, Hernandez J, Supe-Markovina K, D'Alessandri-Silva C, DeJesus-Gonzalez N, Vasylyeva TL, Formeck C, Woll C, Gbadegesin R, Geier P, Devarajan P, Carpenter SL, Kerlin BA, Smoyer WE, Midwest Pediatric Nephrology Consortium (2015) AKI in children hospitalized with nephrotic syndrome. Clin J Am Soc Nephrol 10:2110-2118

19. Baddam S, Aban I, Hilliard L, Howard T, Askenazi D, Lebensburger JD (2017) Acute kidney injury during a pediatric sickle cell vaso-occlusive pain crisis. Pediatr Nephrol 32:14511456

20. Hursh BE, Ronsley R, Islam N, Mammen C, Panagiotopoulos C (2017) Acute kidney injury in children with type 1 diabetes hospitalized for diabetic ketoacidosis. JAMA Pediatr 171:e170020

21. Li S, Krawczeski CD, Zappitelli M, Devarajan P, ThiessenPhilbrook H, Coca SG, Kim RW, Parikh CR, TRIBE-AKI Consortium (2011) Incidence, risk factors, and outcomes of acute kidney injury after pediatric cardiac surgery: a prospective multicenter study. Crit Care Med 39:1493-1499

22. Sutherland SM, Ji J, Sheikhi FH, Widen E, Tian L, Alexander SR, Ling XB (2013) AKI in hospitalized children: epidemiology and clinical associations in a national cohort. Clin J Am Soc Nephrol 8: 1661-1669

23. Hessey E, Morissette G, Lacroix J, Perreault S, Samuel S, Dorais M, Phan V, Jouvet P, Lafrance JP, LeLorier J, Palijan A, Pizzi M, Roy L, Zappitelli M (2018) Healthcare utilization after acute kidney injury in the pediatric intensive care unit. Clin J Am Soc Nephrol 13:685-692

24. Zappitelli M, Moffett BS, Hyder A, Goldstein SL (2011) Acute kidney injury in non-critically ill children treated with aminoglycoside antibiotics in a tertiary healthcare centre: a retrospective cohort study. Nephrol Dial Transplant 26:144-150

25. Kashani K, Rosner MH, Haase M, Lewington AJP, O'Donoghue DJ, Wilson FP, Nadim MK, Silver SA, Zarbock A, Ostermann M, Mehta RL, Kane-Gill SL, Ding X, Pickkers P, Bihorac A, Siew ED, Barreto EF, Macedo E, Kellum JA, Palevsky PM, Tolwani AJ, Ronco C, Juncos LA, Rewa OG, Bagshaw SM, Mottes TA, Koyner JL, Liu KD, Forni LG, Heung M, Wu VC (2019) Quality improvement goals for acute kidney injury. Clin J Am Soc Nephrol 14:941-953

26. Goldstein SL, Dahale D, Kirkendall ES, Mottes T, Kaplan H, Muething S, Askenazi DJ, Henderson T, Dill L, Somers MJG, Kerr J, Gilarde J, Zaritsky J, Bica V, Brophy PD, Misurac J, Hackbarth R, Steinke J, Mooney J, Ogrin S, Chadha V, Warady B, Ogden R, Hoebing W, Symons J, Yonekawa K, Menon S, Abrams L, Sutherland S, Weng P, Zhang F, Walsh K (2020) A prospective multi-center quality improvement initiative (NINJA) indicates a reduction in nephrotoxic acute kidney injury in hospitalized children. Kidney Int 97:580-588

27. Goldstein SL, Kirkendall E, Nguyen H, Schaffzin JK, Bucuvalas J, Bracke T, Seid M, Ashby M, Foertmeyer N, Brunner L, Lesko A, Barclay C, Lannon C, Muething S (2013) Electronic health record identification of nephrotoxin exposure and associated acute kidney injury. Pediatrics 132:e756-e767

28. Goldstein SL, Mottes T, Simpson K, Barclay C, Muething S, Haslam DB, Kirkendall ES (2016) A sustained quality improvement program reduces nephrotoxic medication-associated acute kidney injury. Kidney Int 90:212-221

29. Basu RK, Zappitelli M, Brunner L, Wang Y, Wong HR, Chawla LS, Wheeler DS, Goldstein SL (2014) Derivation and validation of the renal angina index to improve the prediction of acute kidney injury in critically ill children. Kidney Int 85:659-667
30. Xu X, Nie S, Zhang A, Mao J, Liu HP, Xia H, Xu H, Liu Z, Feng S, Zhou W, Liu X, Yang Y, Tao Y, Feng Y, Chen C, Wang M, Zha Y, Feng JH, Li Q, Ge S, Chen J, He Y, Teng S, Hao C, Liu BC, Tang Y, He W, He P, Hou FF (2018) Acute kidney injury among hospitalized children in China. Clin J Am Soc Nephrol 13:1791-1800

31. Kizilbash SJ, Kashtan CE, Chavers BM, Cao Q, Smith AR (2016) Acute kidney injury and the risk of mortality in children undergoing hematopoietic stem cell transplantation. Biol Blood Marrow Transplant 22:1264-1270

32. Bradshaw C, Han J, Chertow GM, Long J, Sutherland SM, Anand S (2019) Acute kidney injury in children hospitalized with diarrheal illness in the United States. Hosp Pediatr 9:933-941

33. Oshomah-Bello EO, Esezobor CI, Solarin AU, Njokanma FO (2020) Acute kidney injury in children with severe malaria is common and associated with adverse hospital outcomes. J Trop Pediatr 66:218-225

34. Krishnamurthy S, Mondal N, Narayanan P, Biswal N, Srinivasan S, Soundravally R (2013) Incidence and etiology of acute kidney injury in southern India. Indian J Pediatr 80:183-189

35. Rajan M, Geminiganesan S, Sankaranarayanan S, Padmanaban R, Selvam MP (2020) Renal manifestations in children with dengue fever hospitalized in pediatric intensive care unit. Indian J Pediatr. https://doi.org/10.1007/s12098-020-03402-z

36. Macedo E, Cerda J, Hingorani S, Hou J, Bagga A, Burdmann EA, Rocco VM, Mehta LR (2018) Recognition and management of acute kidney injury in children: the ISN 0by25 Global Snapshot study. PLoS One 13:e0196586

37. Flynn JT, Kaelber DC, Baker-Smith CM, Blowey D, Carroll AE, Daniels SR, de Ferranti SD, Dionne JM, Falkner B, Flinn SK, Gidding SS, Goodwin C, Leu MG, Powers ME, Rea C, Samuels J, Simasek M, Thaker VV, Urbina EM, Subcommittee on Screening and Management of High Blood Pressure in Children (2017) Clinical practice guideline for screening and management of high blood pressure in children and adolescents. Pediatrics 140: e20171904. https://doi.org/10.1542/peds.2017-1904

38. Dodge WF, West EF, Smith EH, Bruce H 3rd (1976) Proteinuria and hematuria in schoolchildren: epidemiology and early natural history. J Pediatr 88:327-347

39. Hogg RJ, Portman RJ, Milliner D, Lemley KV, Eddy A, Ingelfinger $J(2000)$ Evaluation and management of proteinuria and nephrotic syndrome in children: recommendations from a pediatric nephrology panel established at the National Kidney Foundation conference on proteinuria, albuminuria, risk, assessment, detection, and elimination (PARADE). Pediatrics 105:1242-1249

40. Russell TB, Kram DE (2020) Tumor lysis syndrome. Pediatr Rev 41:20-26

41. Jones GL, Will A, Jackson GH, Webb NJ, Rule S, British Committee for Standards in Haematology (2015) Guidelines for the management of tumour lysis syndrome in adults and children with haematological malignancies on behalf of the British Committee for Standards in Haematology. Br J Haematol 169: 661-671

42. Basu RK, Kaddourah A, Goldstein SL, Akcan-Arikan A, Arnold M, Cruz C, Goldsworthy M, Jaimon N, Alexander S, Festa M, Hahn D, Brown L, Jeon A, Deep A, Askenazi D, Bagshaw S, Morgan C, Alobaidi R, Basu R, Cooper D, Goldstein S, Kaddourah A, Mottes T, Terrell T, Arnold P, Metcalf C, Woodley S, Bogdanović R, Stajić N, Kovacevic B, Peco-Antic A, Paripovic A, Brophy P, Bunchman T, Williams D, Hoot M, Chadha V, Davis K, Dharnidharka V, Walther L, Faustino V, Taft J, Tala J, Gist K, Soranno D, Ha IS, Kang HG, Hackbarth R, Avendt-Reeber M, Butler C, DeGraaf D, Eding D, Hautala N, Ndika A, Hidayati EL, Huang S, Kennedy S, Didsbury M, Kushartono H, Prasetyo R, Mammen C, Paden M, Stone C, Picca S, Connola F, Schneider J, Sweberg T, Kessel A, Selewski D, Hieber S, SpasojevićDimitrijeva B, Ivanisevic I, Putnik J, Ristic S, Sutherland S, 
Staples A, Wong C, Hadid S, Joseph C, Woroniecki R, Zappitelli M, Elsaerafy N, Zaritsky J (2018) Assessment of a renal angina index for prediction of severe acute kidney injury in critically ill children: a multicentre, multinational, prospective observational study. Lancet Child Adolesc Health 2:112-120

43. Basu RK, Chawla LS, Wheeler DS, Goldstein SL (2012) Renal angina: an emerging paradigm to identify children at risk for acute kidney injury. Pediatr Nephrol 27:1067-1078

44. Bhojani S, Stojanovic J, Melhem N, Maxwell H, Houtman P, Hall A, Singh C, Hayes W, Lennon R, Sinha MD, Milford DV, British Association for Paediatric Nephrology (2020) The incidence of paediatric acute kidney injury identified using an AKI e-alert algorithm in six English hospitals. Front Pediatr 8:29

45. Van Driest SL, Wang L, McLemore MF, Bridges BC, Fleming GM, McGregor TL, Jones DP, Shirey-Rice J, Gatto CL, Gay JC, Byrne DW, Weitkamp A, Roden DM, Bernard G (2020) Acute kidney injury risk-based screening in pediatric inpatients: a pragmatic randomized trial. Pediatr Res 87:118-124

46. Goldstein SL, Currier H, Graf C, Cosio CC, Brewer ED, Sachdeva R (2001) Outcome in children receiving continuous venovenous hemofiltration. Pediatrics 107:1309-1312

47. Selewski DT, Cornell TT, Lombel RM, Blatt NB, Han YY, Mottes T, Kommareddi M, Kershaw DB, Shanley TP, Heung M (2011) Weight-based determination of fluid overload status and mortality in pediatric intensive care unit patients requiring continuous renal replacement therapy. Intensive Care Med 37:1166-1173

48. Benoit SW, Goldstein SL, Dahale DS, Haslam DB, Nelson A, Truono K, Davies SM (2019) Reduction in nephrotoxic antimicrobial exposure decreases associated acute kidney injury in pediatric hematopoietic stem cell transplant patients. Biol Blood Marrow Transplant 25:1654-1658

49. Bagshaw SM (2015) Acute kidney injury care bundles. Nephron 131:247-251

50. Logan R, Davey P, Davie A, Grant S, Tully V, Valluri A, Bell S (2018) Care bundles for acute kidney injury: a balanced accounting of the impact of implementation in an acute medical unit. BMJ Open Qual 7:e000392

51. Meijers B, De Moor B, Van Den Bosch B (2016) The acute kidney injury e-alert and clinical care bundles: the road to success is always under construction. Nephrol Dial Transplant 31:1761-1763

52. Schiffl H (2018) Prevention of severe acute kidney injury by implementation of care bundles: some progress but still a lot of work ahead. Saudi J Kidney Dis Transpl 29:513-517

53. Selby NM, Kolhe NV (2016) Care bundles for acute kidney injury: do they work? Nephron 134:195-199

54. Selby NM, Casula A, Lamming L, Stoves J, Samarasinghe Y, Lewington AJ, Roberts R, Shah N, Johnson M, Jackson N, Jones C, Lenguerrand E, McDonach E, Fluck RJ, Mohammed MA, Caskey FJ (2019) An organizational-level program of intervention for AKI: a pragmatic stepped wedge cluster randomized trial. J Am Soc Nephrol 30:505-515

55. Akcan Arikan A, Williams EA, Graf JM, Kennedy CE, Patel B, Cruz AT (2015) Resuscitation bundle in pediatric shock decreases acute kidney injury and improves outcomes. J Pediatr 167:13011305 e1301

56. Gist KM, Selewski DT, Brinton J, Menon S, Goldstein SL, Basu RK (2020) Assessment of the independent and synergistic effects of fluid overload and acute kidney injury on outcomes of critically ill children. Pediatr Crit Care Med 21:170-177

57. Hassinger AB, Wald EL, Goodman DM (2014) Early postoperative fluid overload precedes acute kidney injury and is associated with higher morbidity in pediatric cardiac surgery patients. Pediatr Crit Care Med 15:131-138

58. Sutherland SM, Zappitelli M, Alexander SR, Chua AN, Brophy PD, Bunchman TE, Hackbarth R, Somers MJ, Baum M, Symons JM, Flores FX, Benfield M, Askenazi D, Chand D, Fortenberry JD,
Mahan JD, McBryde K, Blowey D, Goldstein SL (2010) Fluid overload and mortality in children receiving continuous renal replacement therapy: the prospective pediatric continuous renal replacement therapy registry. Am J Kidney Dis 55:316-325

59. Gorga SM, Sahay RD, Askenazi DJ, Bridges BC, Cooper DS, Paden ML, Zappitelli M, Gist KM, Gien J, Basu RK, Jetton JG, Murphy HJ, King E, Fleming GM, Selewski DT (2020) Fluid overload and fluid removal in pediatric patients on extracorporeal membrane oxygenation requiring continuous renal replacement therapy: a multicenter retrospective cohort study. Pediatr Nephrol 35:871882

60. Basu RK, Andrews A, Krawczeski C, Manning P, Wheeler DS, Goldstein SL (2013) Acute kidney injury based on corrected serum creatinine is associated with increased morbidity in children following the arterial switch operation. Pediatr Crit Care Med 14:e218 e224

61. Weiss SL, Peters MJ, Alhazzani W, Agus MSD, Flori HR, Inwald DP, Nadel S, Schlapbach LJ, Tasker RC, Argent AC, Brierley J, Carcillo J, Carrol ED, Carroll CL, Cheifetz IM, Choong K, Cies JJ, Cruz AT, De Luca D, Deep A, Faust SN, De Oliveira CF, Hall MW, Ishimine P, Javouhey E, Joosten KFM, Joshi P, Karam O, Kneyber MCJ, Lemson J, MacLaren G, Mehta NM, Moller MH, Newth CJL, Nguyen TC, Nishisaki A, Nunnally ME, Parker MM, Paul RM, Randolph AG, Ranjit S, Romer LH, Scott HF, Tume LN, Verger JT, Williams EA, Wolf J, Wong HR, Zimmerman JJ, Kissoon N, Tissieres P (2020) Surviving sepsis campaign international guidelines for the management of septic shock and sepsisassociated organ dysfunction in children. Pediatr Crit Care Med 21: e52-e106

62. Weiss SL, Peters MJ, Alhazzani W, Agus MSD, Flori HR, Inwald DP, Nadel S, Schlapbach LJ, Tasker RC, Argent AC, Brierley J, Carcillo J, Carrol ED, Carroll CL, Cheifetz IM, Choong K, Cies JJ, Cruz AT, De Luca D, Deep A, Faust SN, De Oliveira CF, Hall MW, Ishimine P, Javouhey E, Joosten KFM, Joshi P, Karam O, Kneyber MCJ, Lemson J, MacLaren G, Mehta NM, Moller MH, Newth CJL, Nguyen TC, Nishisaki A, Nunnally ME, Parker MM, Paul RM, Randolph AG, Ranjit S, Romer LH, Scott HF, Tume LN, Verger JT, Williams EA, Wolf J, Wong HR, Zimmerman JJ, Kissoon N, Tissieres P (2020) Executive summary: surviving sepsis campaign international guidelines for the management of septic shock and sepsis-associated organ dysfunction in children. Pediatr Crit Care Med 21:186-195

63. Weiss SL, Peters MJ, Alhazzani W, Agus MSD, Flori HR, Inwald DP, Nadel S, Schlapbach LJ, Tasker RC, Argent AC, Brierley J, Carcillo J, Carrol ED, Carroll CL, Cheifetz IM, Choong K, Cies JJ, Cruz AT, De Luca D, Deep A, Faust SN, De Oliveira CF, Hall MW, Ishimine P, Javouhey E, Joosten KFM, Joshi P, Karam O, Kneyber MCJ, Lemson J, MacLaren G, Mehta NM, Moller MH, Newth CJL, Nguyen TC, Nishisaki A, Nunnally ME, Parker MM, Paul RM, Randolph AG, Ranjit S, Romer LH, Scott HF, Tume LN, Verger JT, Williams EA, Wolf J, Wong HR, Zimmerman JJ, Kissoon N, Tissieres P (2020) Surviving sepsis campaign international guidelines for the management of septic shock and sepsisassociated organ dysfunction in children. Intensive Care Med 46: $10-67$

64. Weiss SL, Peters MJ, Alhazzani W, Agus MSD, Flori HR, Inwald DP, Nadel S, Schlapbach LJ, Tasker RC, Argent AC, Brierley J, Carcillo J, Carrol ED, Carroll CL, Cheifetz IM, Choong K, Cies JJ, Cruz AT, De Luca D, Deep A, Faust SN, De Oliveira CF, Hall MW, Ishimine P, Javouhey E, Joosten KFM, Joshi P, Karam O, Kneyber MCJ, Lemson J, MacLaren G, Mehta NM, Moller MH, Newth CJL, Nguyen TC, Nishisaki A, Nunnally ME, Parker MM, Paul RM, Randolph AG, Ranjit S, Romer LH, Scott HF, Tume LN, Verger JT, Williams EA, Wolf J, Wong HR, Zimmerman JJ, Kissoon N, Tissieres P (2020) Executive summary: surviving sepsis campaign international guidelines for the management of septic 
shock and sepsis-associated organ dysfunction in children. Intensive Care Med 46:1-9

65. Mottes TA, Goldstein SL, Basu RK (2019) Process based quality improvement using a continuous renal replacement therapy dashboard. BMC Nephrol 20:17

66. Mottes T, Owens T, Niedner M, Juno J, Shanley TP, Heung M (2013) Improving delivery of continuous renal replacement therapy: impact of a simulation-based educational intervention. Pediatr Crit Care Med 14:747-754

67. Hackbarth R, Bunchman TE, Chua AN, Somers MJ, Baum M, Symons JM, Brophy PD, Blowey D, Fortenberry JD, Chand D, Flores FX, Alexander SR, Mahan JD, McBryde KD, Benfield MR, Goldstein SL (2007) The effect of vascular access location and size on circuit survival in pediatric continuous renal replacement therapy: a report from the PPCRRT registry. Int J Artif Organs 30:1116-1121
68. Kidney Disease: Improving Global Outcomes (KDIGO) Acute Kidney Injury Work Group (2012) KDIGO clinical practice guideline for acute kidney injury. Kidney Int Suppl 2:1-138

69. Kellum JA (2015) Persistent acute kidney injury. Crit Care Med 43: 1785-1786

70. Hoste E, Bihorac A, Al-Khafaji A, Ortega LM, Ostermann M, Haase M, Zacharowski K, Wunderink R, Heung M, Lissauer M, Self WH, Koyner JL, Honore PM, Prowle JR, Joannidis M, Forni LG, Kampf JP, McPherson P, Kellum JA, Chawla LS, Investigators RUBY (2020) Identification and validation of biomarkers of persistent acute kidney injury: the RUBY study. Intensive Care Med 46:943-953

Publisher's note Springer Nature remains neutral with regard to jurisdictional claims in published maps and institutional affiliations. 